\title{
Affidavit présenté dans le cadre de la plainte devant la Cour fédérale du Commissaire aux langues officielles du Canada visant les compressions budgétaires de CBEF Windsor
}

\author{
Simon Laflamme
}

Volume 12, 2018

URI : https://id.erudit.org/iderudit/1048915ar

DOI : https://doi.org/10.7202/1048915ar

Aller au sommaire du numéro

Éditeur(s)

Société Charlevoix

Presses de l’Université d'Ottawa

ISSN

1203-4371 (imprimé)

2371-6878 (numérique)

Découvrir la revue

Citer cet article

Laflamme, S. (2018). Affidavit présenté dans le cadre de la plainte devant la Cour fédérale du Commissaire aux langues officielles du Canada visant les compressions budgétaires de CBEF Windsor. Cahiers Charlevoix, 12, 13-54. https://doi.org/10.7202/1048915ar
Résumé de l'article

L'affidavit que publie ici Simon Laflamme a été utilisé par le Commissariat aux langues officielles pour appuyer la cause qu'il défendait contre la Société Radio-Canada. À la suite des compressions budgétaires de 2009 dont elle avait été victime, la société d'État réduisait fortement sa programmation à la station de radio $\mathrm{CB} \mathrm{F}$ de Windsor. Ce document rappelle quelques principes sur le rapport entre les communautés et leurs médias en insistant sur les conditions de la postmodernité et sur la situation des populations minoritaires, notamment sur celle de la francophonie canadienne; il distingue le rôle des médias généralistes de celui des médias spécialisés en insistant sur l'importance pour une population donnée de produire des messages médiatiques pour elle-même ; il met en évidence la dynamique entre démographie, culture, médias et économie ; il évoque une série de travaux empiriques sur les médias qui permettent aussi bien de comprendre le cas franco-ontarien dans son ensemble que de saisir la spécificité de la communauté francophone du sud-ouest de la province. 


\section{Affidavit présenté dans le cadre de la plainte devant la Cour fédérale du Commissaire aux langues officielles du Canada visant les compressions budgétaires de CBeF Windsor}

Simon LAFLAMme

Département de sociologie Université Laurentienne 


\section{SOMMAIRE}

EXPERTISE

PRINCIPES GÉNÉRAUX 18

Prémodernité, modernité et postmodernité 18

Différenciation et homogénéisation 22

Postmodernité et hétérogénéité 23

Majorité et minorité $\quad 23$

La langue comme caractéristique des communautés $\quad 26$

Médias généralistes et médias spécialisés $\quad 27$

Production et diffusion des messages 28

Économie, démographie, culture et médias $\quad 29$

L'importance des médias pour les communautés $\quad 32$

Des médias pour la communauté $\quad 36$

ONTARIO FRANÇAIS ET FRANCOPHONIE MINORITAIRE AU CANADA 37

Une minorité importante dans des situations différentes $\quad 37$

Une minorité qui se compare à la majorité $\quad 38$

Une minorité qui dispose de la même technologie médiatique $\quad 40$

Une puissante anglicité $\quad 41$

Instruction et rapport linguistique aux médias $\quad 45$

LES FRANCOPHONES DU SUD-OUEST DE L'ONTARIO 48

$\begin{array}{ll}\text { CONCLUSION } & 51\end{array}$ 


\section{Affidavit présenté dans le cadre de la plainte devant la Cour fédérale du Commissaire aux langues officielles du Canada visant les compressions budgétaires de Cbef Windsor}

En 2009, le Gouvernement fédéral sabre dans le budget de Canadian Broadcasting Corporation / Société Radio-Canada en y soustrayant 171 millions de dollars. Radio-Canada choisit alors de réduire son personnel, de modifier sa programmation et de vendre des actifs. La station de radio CBEF de Windsor est fortement affectée par les compressions. Des francophones du sud-ouest de l'Ontario, le docteur Karim Amellal en tête, font entendre leurs récriminations au Commissaire aux langues officielles et au Conseil de la radiodiffusion et des télécommunications canadiennes (CRTC). Le Commissaire aux langues officielles approche Radio-Canada pour relayer les inquiétudes exprimées par la minorité francophone. Radio-Canada soutient qu'elle n'a pas de compte à rendre au Commissaire, qu'elle n'est pas soumise à la Loi sur les langues officielles sinon indirectement dans la programmation qui est réglementée par le CRTC. Le CRTC est invité à réagir, mais il ne le fait pas. La cause du Commissaire est déposée devant la Cour supérieure de l'Ontario qui se déclare incompétente. Elle se déplace devant une Cour fédérale ${ }^{1}$. Le 29 mai 2012, le juge Luc Martineau, dans une décision interlocutoire, suspend provisoirement les procédures. L'intention est que RadioCanada et le Commissaire en viennent à s'entendre entre eux, qu'ils trouvent une solution conjointement avec le CRTC ${ }^{2}$. Les

1. $\mathrm{N}^{\circ}$ du dossier de la Cour : T-1288-10.

2. Cour fédérale, référence 2012 CF 650, Ottawa (Ontario), 29 mai 2012, http://www.cbc.radio-canada.ca/_files/cbcrc/documents/proactive-disclosure/ 
positions ne se réconcilieront pas, on ne fera rien pour corriger la situation. Conscient de la complexité du litige et de l'intensité de la divergence des convictions, le 8 mars 2014, le juge Martineau « prononce la suspension permanente des procédures ${ }^{3}$ ».

Pour légitimer son propos, le Commissariat aux langues officielles a sollicité quelques opinions. C'est l'une d'elles que nous donnons ici à lire ${ }^{4}$. Dans cet affidavit ${ }^{5}$, nous rappelons, bien sûr, la thèse de la complétude institutionnelle. L'essentiel de notre argumentation, cependant, repose sur trois principes. Le premier est celui de la transformation des sociétés, depuis la prémodernité jusqu'à la postmodernité, et du rôle prépondérant que jouent les médias de masse, et tout particulièrement la radio, dans la modernité et dans la postmodernité. Le deuxième est celui de la vulnérabilité de la francophonie minoritaire au Canada et tout spécialement de celle du sud-ouest de l'Ontario. Le troisième est celui de la responsabilité de la radio d'État. Tout au long de cet exposé, nous insistons sur la dialectique qui, dans toute société, génère des mouvements vers la différenciation et vers l'homogénéisation. L'évocation de cette tension permet de bien saisir l'individualisation et la collectivisation du rapport aux médias et de comprendre l'importance de la collectivisation francophone du sud-ouest au cœur du mouvement vers l'homogénéisation dont l'un des principaux moteurs est la diffusion de messages médiatiques anglophones, notamment étatsuniens.

Le procès que le Commissaire aux langues officielles a intenté contre Radio-Canada n'a pas connu la victoire espérée. Mais il

judgements/2012-05-29-decision-juge-martineau.pdf, site consulté en mars 2018.

3. Cour fédérale, référence 2014 CF 849, Ottawa (Ontario), 8 septembre 2014, http://cas-cdc-www02.cas-satj.gc.ca/rss/T-1288-10\%20decision\%20FR\%20FINAL. pdf, site consulté en mars 2018.

4. Affidavit de Simon Laflamme (Règle 80 des Règles des Cours fédérales), Cour fédérale entre le Commissaire aux langues officielles du Canada, demandeur, et CBC/Radio-Canada, défenderesse, $\mathrm{n}^{\circ}$ du dossier de la Cour T-01288-10, septembre 2010.

5. On trouvera sans doute des similitudes dans le geste entre ce texte qui a été écrit au bénéfice de la radio francophone de Windsor et l'affidavit que Roger Bernard a écrit contre la fermeture d'un hôpital francophone et qui a été publié sous le titre $\dot{A}$ la défense de Montfort (Ottawa, Le Nordir, 2001). 
n'a pas été vain. La programmation de CBEF cesse en juin 2009, mais elle reprend progressivement à partir de septembre 2010. L'importante émission Matins sans frontières reprend alors l'antenne pour une durée d'une heure, puis à compter de mai 2013, pour deux heures, puis, au cours de l'été 2015, pour trois heures, de 6 h 00 à 9 h 00, du lundi au vendredi.

$* * *$

Je soussigné, Simon Laflamme, de la ville du Grand Sudbury, dans la province de l'Ontario, affirme solennellement que :

1. Je suis docteur en sociologie, expert en sociologie de la communication et des médias.

2-5. Dans le document original, ces paragraphes 2 à 5 contiennent des informations d'ordre général sur la formation et la profession qu'on trouve dans le curriculum vitae.

\section{EXPERTISE}

6. J'ai été approché par les procureurs du Commissaire aux langues officielles du Canada afin de présenter un avis d'expert dans le présent dossier. Pour ce faire, j'ai pris connaissance du rapport d'enquête final du Commissaire aux langues officielles concernant les plaintes visant $\mathrm{CBC} /$ Radio-Canada et portant sur des compressions budgétaires effectuées par $\mathrm{CBC} /$ Radio-Canada à la station de radio $\mathrm{CBEF}$ de Windsor.

7. Dans ce texte, j'entends faire valoir l'importance des médias dans les sociétés postmodernes et démontrer en quoi ces médias sont nécessaires tout particulièrement aux minorités qui font partie de ces sociétés. Pour y parvenir, j'énumère un ensemble de principes qui sont attachés les uns aux autres. À travers cette énumération, je montre à quel point la radio est utile aux diverses populations par l'immanence de son ancrage dans une population et par son aptitude à s'inscrire pratiquement dans la quotidienneté des événements. Cette énumération faite, dans un deuxième temps, je présente le cas de la communauté franco-ontarienne et, plus généralement, 
celui de la minorité francophone du Canada ; j'insiste alors sur les comportements linguistiques de cette minorité dans ses rapports aux médias. Enfin, dans un troisième temps, je m'arrête sur la situation des francophones du sud-ouest de la province ontarienne ; je montre que le recours de la population au français dans les médias dépend fortement de l'âge, que les plus jeunes tendent à se détourner du français et que les adultes l'apprécient. Pour ces deux raisons opposées, je conclus qu'il importe de maximiser là l'offre de messages médiatiques en français, notamment celle qui provient de la radio.

\section{Principes GÉnérauX}

\section{Prémodernité, modernité et postmodernité}

8. On dit de bon nombre de sociétés contemporaines qu'elles sont postmodernes. Cela signifie que, dans ces sociétés, les facteurs de détermination prémodernes et modernes ne jouent plus les rôles qu'ils jouaient et que, par conséquent, les traditions se reproduisent difficilement ${ }^{6}$.

\section{La prémodernité}

9. Dans les sociétés prémodernes, les institutions familiale et religieuse définissent fortement les comportements des individus. La vie communautaire y est intense de sorte que l'essentiel des rapports sociaux est constitué de relations interpersonnelles in praesentia. Dans ces relations, l'individu apprend ce qui caractérise sa communauté et reproduit par ses actions et son discours l'essence de cette communauté ; il reproduit ainsi, par exemple, les modes familial et religieux.

6. Frédéric Barbier et Catherine Bertho Lavenir, Histoire des médias de Diderot à Internet, Paris, Armand Colin, 1996 ; Philippe Breton et Serge Proulx, L'Explosion de la communication à l'aube du XXI siècle, Montréal, Boréal / Paris, La Découverte, [1989] 2002 ; Manuel Castells, The Rise of the Network Society, vol. 1, The Information Age : Economy, Society and Culture, Oxford, Blackwell, 1996 ; Sylvie Douzou et Kevin G. Wilson, Une histoire des médias de communication, Québec, Téléuniversité, [1994] 2009 ; Mélanie Girard et Simon Laflamme, « La Sociologie de la communication », dans Jean Lafontant et Simon Laflamme (dir.), Initiation thématique à la sociologie, Sudbury, Prise de parole, « Cognitio », 2008, p. 427-452; Éric Maigret, Sociologie de la communication et des médias, Paris, Armand Colin, 2003. 
La dominance du travail paysan, et donc la similitude des tâches auxquelles s'adonnent les individus pour assurer leur survie, facilite cette reproduction. Le changement, dans ces sociétés, est normalement impulsé par des facteurs externes comme des disettes ou des contacts avec d'autres populations.

\section{La modernité}

10. Dans les sociétés modernes, l'ensemble des déterminants s'étend quelque peu. À la famille et la religion s'ajoutent, par exemple, l'école et l'industrialisation dont le corollaire est l'urbanisation. Les relations interpersonnelles continuent de jouer un rôle majeur dans la reproduction des modes traditionnels, et donc des formes de la vie sociale. L'école établit des liens entre les diverses communautés qui composent la société en transmettant des savoirs communs aussi bien techniques que moraux, et même, plus généralement, idéologiques. Par conséquent, même si la société connaît une expansion, les relations interpersonnelles, sous l'effet des institutions, rendent possibles l'apparition et la reproduction de formes communes de socialité. L'incidence des médias commence à se faire sentir. Le livre, puis le journal, puis la radio permettent à un nombre de plus en plus grand d'individus de s'exposer à un même message. Grâce à ces médias, la société pourra se densifier et s'étendre tout en assurant en elle une certaine homogénéité du discours, et donc de la conscience collective. L'apport des médias est ainsi fondamental, mais celui des institutions comme la famille et la religion ne l'est pas moins. Les relations interpersonnelles restent fortes malgré l'effet des rapports médiatisés. Les forces centripètes et homogénéisantes de la culture sont dominantes. Elles le sont malgré le fait d'une division du travail qui, notamment, voit apparaître un espace ouvrier croissant et composite.

\section{La postmodernité}

11. Dans les sociétés postmodernes, la famille, la religion, l'école, 
le travail continuent d'avoir une influence sur les individus et sur les formes de la vie en société. Mais le système des déterminants se complexifie.

12. Les sources d'influence deviennent plus nombreuses en vertu d'un processus de subdivision. Les structures familiales se font plus nombreuses. Plusieurs coexistent dans une société spécifique ; un enfant peut avoir vécu ou même vivre par alternance dans plus d'une de ces structures ; un adulte peut passer de l'une à l'autre ou même passer d'un ménage qui compte plusieurs personnes à un autre où il sera seul. L'univers religieux se pluralise. Bon nombre de religions se proposent à un même individu, simultanément ou successivement. La religion donne lieu à des réflexions qui peuvent conduire à des fabrications individualisées ou à des expressions d'athéisme, tout comme elle peut conduire à l'adulation collective d'un prêcheur. L'école offre des formations et des niveaux de plus en plus nombreux. Elle propose même des enseignements adaptés à divers milieux ou à divers ensembles d'individus. L'univers du travail non seulement n'est plus à dominance paysanne ou ouvrière, mais en plus il est caractérisé par une infinité de tâches, qui supposent une multitude de compétences, de même que par de nombreuses transformations.

13. La complexification du système de détermination se révèle surtout dans la pluralisation des formes de la circulation de l'information. Cette pluralisation a pour corollaire l'expansion et la variation du champ médiatique. Dans les sociétés prémodernes, l'essentiel de l'information qui circule est défini par les interactions entre les personnes qui se côtoient physiquement et par la similitude des actions qui ont cours. La reproduction du même est donc très probable. Dans les sociétés modernes, la division du travail et la densification des populations tendent à induire une diversification de l'information, mais ce phénomène est fortement atténué par l'importance des échanges entre des personnes physiques qui appartiennent à des communautés spécifiques, par le rôle de 
l'école et de la religion et par l'effet des médias de masse. La faible tendance à l'hétérogénéisation de l'information est freinée par la forte tendance à l'homogénéisation. La tradition parvient donc à se pérenniser malgré des forces antinomiques. Dans les sociétés postmodernes, un individu particulier est exposé à des informations variées attribuables à la subdivision des sources d'influence sociale, certes, mais surtout au fait que le champ médiatique lui offre des messages de masse variés, voire contradictoires, et que le rapport aux médias eux-mêmes peut se présenter selon une infinitude de combinaisons. Une même personne est exposée à de nombreux contenus parce qu'un même média livre une diversité d'informations et que les médias eux-mêmes sont nombreux à livrer des informations. Le rapport qu'une personne entretient avec ces contenus et ces médias est lui-même diversifié. La circulation de l'information dépend ainsi, moins que dans les sociétés prémodernes et modernes, des relations in praesentia avec d'autres individus et des encadrements institutionnels. Cela donne beaucoup de poids aux forces hétérogénéisantes de la société. Ce que l'enfant entend de la bouche de ses parents peut très bien être contredit par ce qui est exprimé dans les messages médiatiques que reçoit l'enfant ou par ce qui est véhiculé par ses amis ou par les parents de ses amis.

14. Que la société soit prémoderne, moderne ou postmoderne, les messages qui sont émis ne suivent pas une trajectoire unilatérale. Les individus ne sont pas de simples réceptacles des messages qu'ils perçoivent. Ils donnent forme à ces messages par un travail intérieur et en fonction des relations qu'ils entretiennent avec les autres sources émettrices. Les messages qui circulent agissent sur les individus, qui eux-mêmes agissent sur les messages. Dans les sociétés postmodernes, cette dialectique est décuplée par l'ouverture du champ médiatique et par la subdivision des autres sources d'influence. Cela contribue à étendre le domaine des messages qui circulent socialement. 
15. L'interaction entre un champ médiatique pluriel et des sources subdivisibles d'influence sociale combinée à une influence réciproque des messages et des individus ouvrent l'univers de l'information qui appartient à une société postmoderne sur l'hétérogénéité.

\section{Différenciation et homogénéisation}

16. Dans toutes les sociétés, il y a des forces de différenciation et d'homogénéisation. Aucune société ne peut être pure uniformité ou absolue dissimilitude. Dans la société prémoderne, la similitude est grande ; mais elle n'empêche pas des différences entre les âges ou les sexes, par exemple, de même que des agissements individuels. Dans la société postmoderne, les comportements individuels sont innombrables, mais ils n'abolissent pas les modes communs de pensée ou d'agir. Ce qui distingue la société postmoderne de la société prémoderne, ce n'est pas la tension entre différenciation et indifférenciation, c'est l'envergure de cette tension. Les sociétés postmodernes, en effet, occupent souvent des territoires étendus où les rapports interindividuels et même collectifs in praesentia sont impossibles; elles sont communément populeuses et diversifiées. En l'absence de forces médiatiques, elles ne parviendraient pas à générer la similitude qui leur est nécessaire, à réunir les populations autour de messages communs, à assurer les interactions entre les individus qui ont quelque chose en partage en dépit de la distance qui les sépare et de la multiplicité des lieux qu'ils occupent ${ }^{7}$.

7. Vittorio Cotesta, Images du monde et société globale : grandes interprétations et débats actuels, Sainte-Foy, Presses de l'Université Laval, 2006 ; Philippe d'Iribarne, La Logique de l'honneur : gestion des entreprises et traditions nationales, Paris, Seuil, 1989 ; Philippe d'Iribarne, Alain Henry, Jean-Pierre Segal et Sylvie Chevrier, Culture et mondialisation. Gérer par-delà les frontières, Paris, Seuil, [1998] 2002 ; Roger Gervais, «Presse et mondialisation : étude comparée franco-canadienne », thèse pour le doctorat en sociologie délivré par l'Université des Sciences Sociales de Toulouse I, 2009 ; Simon Laflamme et Ali Reguigui, Homogénéité et distinction, Sudbury, Prise de parole, 2003 ; David Robinson, Muslim Societies in African History, Cambridge, Cambridge University Press, 2004. 


\section{Postmodernité et hétérogénéité}

17. Les sociétés postmodernes sont marquées par la diversité comme fait et la diversification comme processus. Cette hétérogénéisation se révèle dans les pensées et les actions des individus, mais elle se manifeste aussi en fonction de formes collectives. Une société postmoderne comporte souvent plusieurs ethnies caractérisées, par exemple, par la langue ou la religion. Ces ethnies peuvent elles-mêmes être marquées par la diversité et occuper des lieux différents sur un territoire donné. Les diverses ethnies qui composent une société peuvent ou non vivre en harmonie. Mais elles ont toutes besoin de médias pour se reproduire dès lors qu'elles ont une certaine population. Dans la postmodernité, il n'y pas de communauté ethnique socialement étendue qui puisse se reproduire en l'absence de médias ${ }^{8}$.

18. Toutes les communautés qui composent une société ne sont pas du même ordre et ne procèdent pas du même rapport à la société dans son ensemble. Généralement, la démographie et l'histoire constituent les principaux facteurs de différenciation de ces communautés. Sauf exception, celles qui sont les plus populeuses et qui, symboliquement, définissent le plus l'histoire de la société sont celles dont le statut est le moins contesté. Ces communautés tendent d'ailleurs à se doter de médias ou à en réclamer.

\section{Majorité et minorité}

19. On distingue communément les sociétés selon qu'elles sont majoritaires ou minoritaires. Dans la plupart des cas, le majoritaire est effectivement plus nombreux que le minoritaire. Mais, par étirement de sens, on associe souvent majorité à

8. Yves Boisvert, Le Monde postmoderne : analyse du discours sur la postmodernité, Paris, L'Harmattan, « Logiques sociales »,1996 ; Yves Boisvert (dir.), Postmodernité et sciences humaines. Une notion pour comprendre notre temps, Montréal, Liber, 1998 ; Anthony Giddens, The Consequences of Modernity, Cambridge, Polity, 1990 ; Anthony Giddens, The Modernity and Self-Identity. Self and Society in the Late Modern Age, Cambridge, Polity, 1991 ; Jeffrey G. Reitz (dir.), Globalization and Society. Process of Differenciation Examined, Westport, Praeger, 2003. 
privilège, de même que minorité à marginalisation; dans cet esprit, on peut comprendre qu'une population qui aurait un statut socialement enviable serait considérée comme majoritaire même si elle était moins nombreuse que la population qui serait en situation d'infériorité par rapport à elle.

20. La sociologie observe que, généralement, une communauté majoritaire comprend plus d'individus qu'une communauté minoritaire et que, sur le plan sociétal, elle jouit d'un ascendant, souvent à plusieurs niveaux, par exemple politique, symbolique, économique, médiatique, éducatif. Plus cet ascendant est élevé, plus les communautés minoritaires sont menacées. Elles le sont de deux façons. Elles le sont d'abord parce que le réseau institutionnel de la minorité est défavorisé et que, par conséquent, il lui est difficile de se reproduire. En effet, pour se faire être, depuis la modernité, une communauté a besoin de toutes les institutions qui font que sa population peut réellement s'inscrire dans la socialité : il lui faut un système éducatif complet, des lieux de pratique religieuse, des centres récréatifs, des centres culturels, des événements publics, des lieux de travail, des médias. Elles le sont ensuite parce que la position du majoritaire, corroborée continuellement par la place de ses institutions dans l'ensemble sociétal, représente un fort pouvoir d'attraction.

21. Les systèmes institutionnels sont fondamentaux pour le devenir aussi bien du minoritaire que du majoritaire 9

22. Les systèmes institutionnels constituent des ensembles intégrés qui font que l'individu qui appartient à une communauté peut, en principe, de façon significative, vivre en elle au quotidien, et jusqu'à sa mort. Ils constituent aussi

9. Réal Allard, Rodrigue Landry et Kenneth Deveau, « La Vitalité ethnolinguistique et l'étude du développement bilingue des minorités acadienne et francophones du Canada atlantique », dans André Magord (dir.), Adaptation et innovation. Expériences acadiennes contemporaines, Bruxelles, P.I.E.-Peter Lang, 2006, p. 85-104 ; Raymond Breton, «Institutional Completeness of Ethnic Communities and the Personal Relations of Immigrants », American Journal of Sociology, no 70, 1964, p. 193-205 ; Rodrigue Landry, Réal Allard et Kenneth Deveau, « Revitalisation ethnolinguistique. Un modèle macroscopique », dans André Magord (dir.), op. cit., p. 105-124. 
des réseaux, car, dans les sociétés postmodernes, une même communauté occupe divers lieux. Écoles, églises et médias, par exemple, doivent être partout où la communauté s'est établie. En outre, ces réseaux doivent permettre à la communauté de se reproduire aussi bien dans ses particularismes, ce qui suppose une forte autonomie des institutions locales, que dans son ensemble, ce qui implique la circulation de messages collectifs qui déborde le cadre des lieux spécifiques.

23. Ainsi, plus la communauté minoritaire dispose d'institutions et plus ces institutions lui permettent de s'inscrire dans la tension nécessaire entre différenciation et homogénéisation, alors plus elle est à même de se reproduire. Car moins est dommageable la différence entre le majoritaire et le minoritaire. Une communauté a besoin d'écoles pour se reproduire, mais elle ne peut pas se reproduire si elle a pour seule institution des écoles. Dans la postmodernité, l'apport des médias est aussi déterminant que celui de l'école ou de la famille, ou de la religion, ou du milieu de travail ${ }^{10}$.

24. Mais le statut d'une communauté au sein de la société ne lui vient pas de sa seule position interne. Entrent en ligne de compte des considérations extérieures. Si le majoritaire, par

10. Donald Browne, Electronic Media and Indigenous Peoples : A Voice of Our Own ?, Ames (IA), Iowa State University Press, 1996 ; Daniel Cunliffe, " Minority Languages and the Internet: New Threats, New Opportunities », dans Mike Cormack et Niamh Hourigan, Minority Language Media. Concepts, Critiques and Case Studies, Cleveland, Buffalo et Toronto, Multilingual Matters, 2007, p. 133-150 ; Fernand Harvey (dir.), Médias francophones hors Québec et identité. Analyses, essais et témoignages, Québec, Institut québécois de recherche sur la culture, 1992 ; Simon Laflamme, Nicole Corbett et Chris Southcott, «Usages des médias et langue de communication dans la communauté francophone du nord-ouest de l'Ontario », Revue du Nouvel-Ontario, n 33, 2008, p. 69-94 ; Rodrigue Landry, « Socialisation par les médias et l'identité », communication présentée dans le cadre du Congrès international 2009 de l'Association de la presse francophone, Moncton, 2009 ; Tom Moring, « Functional Completeness in Minority Language Media », dans Mike Cormack et Niamh Hourigan, Minority Language Media. Concepts, Critiques and Case Studies, Cleveland, Buffalo et Toronto, Multilingual Matters, 2007, p. 17-33 ; Eithne O’Connell, « Translation and Minority Language Media: Potential and Problems : An Irish Perspective », dans Mike Cormack et Niamh Hourigan, Minority Language Media. Concepts, Critiques and Case Studies, Cleveland, Buffalo et Toronto, Multilingual Matters, 2007, p. 212-228 ; Sari Pietikäinen, «Broadcasting Indigenous Voices : Sami Minority Media Production», European Journal of Communication, vol. 23, nº 2, 2008, p. 173-191 ; Stephen H. Riggins (dir.), Etnnic Minority Media : An International Perspective, Londres, Sage, 1992. 
certains de ses traits, appartient à un ensemble dont la place est internationalement importante, alors sa position de majoritaire est d'autant consolidée. Si le minoritaire a quelque similarité avec des populations qui sont mondialement valorisées, alors son statut de minoritaire est atténué.

\section{La langue comme caractéristique des communautés}

25. Le minoritaire peut se distinguer du majoritaire selon divers critères. Les plus usuels sont d'ordre ethnique. Dans les sociétés postmodernes, il s'agit surtout de la langue et de la religion.

26. Quel que soit le facteur qui distingue le minoritaire du majoritaire, les deux communautés ont besoin de médias.

27. Le critère peut être essentiellement religieux. Si tel est le cas, il n'y a pas de barrière linguistique entre les deux communautés. En fonction de l'ascendant sociétal du majoritaire et dans la mesure où les messages médiatiques du majoritaire ne sont pas religieux, l'attention des individus qui appartiennent à la communauté minoritaire tendra à se déplacer vers les messages médiatiques de la communauté majoritaire. Ces passages sont d'autant moins préjudiciables à la communauté minoritaire que les médias livrent des contenus non religieux.

28. Si le critère est essentiellement linguistique, il n'en va pas pareillement. En effet, si le minoritaire s'expose aux médias du majoritaire, il met alors de côté le trait qui lui est le plus caractéristique. Certes, il n'y pas d'identité sociale qui soit strictement linguistique. À travers des langues différentes peuvent s'exprimer des symboliques comparables. Ainsi, l'individu qui appartient à une minorité linguistique qui s'expose aux messages médiatiques de la majorité peut trouver des contenus qui correspondent à son éthos. Mais il n'en demeure pas moins qu'un média peut difficilement ne pas livrer un contenu linguistique. Puisqu'il en est ainsi, que la frontière est souvent poreuse entre les contenus médiatiques de la population minoritaire et ceux de la population majo- 
ritaire et que la majorité jouit communément d'un capital de sympathie dans l'esprit du minoritaire, le glissement vers les messages du majoritaire est aisé et il n'a ni plus ni moins pour conséquence que la fragilisation du minoritaire.

29. En soi, l'exposition, pour une population donnée, aux messages que livre le système médiatique d'une autre population ne représente pas une menace pour la première. Cela peut même représenter une source d'enrichissement de sa culture. Le danger vient de ce que cette exposition ait lieu parce que les messages médiatiques font défaut; si tel est le cas, dans la postmodernité, les forces assimilatrices de la population qui livre les messages auxquels on s'expose s'avèrent infiniment grandes. Le danger vient aussi du fait que l'exposition aux messages de l'autre, surtout en contexte de dynamique linguistique, soit essentiellement unilatérale. Ce sont davantage les minoritaires qui comprennent la langue de l'autre que les majoritaires, ce qui a pour effet que l'attrait des messages du majoritaire soit plus manifeste que celui des messages du minoritaire et que, par conséquent, c'est beaucoup plus le minoritaire que le majoritaire qui ait à en craindre les effets négatifs sur sa communauté.

\section{Médias généralistes et médias spécialisés}

30. Il y a des médias généralistes et des médias spécialisés. Les premiers livrent des contenus variés où se côtoient selon diverses proportions de l'information et du divertissement ; ils s'adressent à un public diversifié. Les seconds offrent des messages ciblés destinés à des publics définis. Ce sont surtout les médias généralistes qui assurent la circulation de l'information dans les communautés. En fait, on trouve d'autant plus de médias spécialisés, dans une société donnée, que les médias généralistes sont bien implantés en elle.

31. C'est très souvent vers les médias généralistes que se tourne une communauté pour savoir ce qui se passe en elle à maints égards et pour y trouver sa propre image. C'est auprès d'une 
radio généraliste, par exemple, que s'informe une population pour savoir ce qui est arrivé la veille, ce qui aura lieu aujourd'hui, quelle est la température, quel est l'état de la circulation, pour entendre une chanson, écouter un invité, et ainsi de suite.

32. Les médias généralistes sont de deux types. Il y a ceux qui s'adressent à une société ou à une communauté dans son ensemble, indépendamment, par exemple, de la spécificité des lieux où habitent les destinataires des messages. Il y a aussi ceux qui ont pour destinataire une communauté régionalement située. Ces médias émettent des messages qui sont susceptibles d'intéresser une population en fonction de sa spécificité régionale. Pour se faire être, une communauté doit aussi bien s'exposer aux contenus qui intéressent la communauté dans son ensemble qu'à ceux qui s'adressent à elle de manière spécifique. En l'absence de cette dualité, il lui est difficile de se reproduire entre la différenciation et l'homogénéisation. Mais pour que cette dualité soit réelle, cela suppose aussi bien un accès à des contenus transcommunautaires qu'à des contenus particuliers. Dans la concurrence des médias, si le communautaire spécifique est trop périphérique dans l'ensemble des messages que diffuse un média, alors les membres de la communauté vont se tourner vers d'autres médias.

\section{Production et diffusion des messages}

33. Pour être médiatisé, un message doit être produit et diffusé. Cela suppose une infrastructure économique. Dans le cas de contenus cinématographiques ou télévisuels, normalement, cela suppose une grande capitalisation, surtout quand il s'agit de contenus catégorisés comme divertissants, qu'on distingue généralement des contenus informatifs.

34. Sur le plan sociétal, une population, pour pouvoir se doter de messages, doit être en mesure non seulement de se pourvoir d'infrastructures médiatiques qui rendent possibles aussi bien la production de contenus que leur diffusion et leur réception, 
mais encore de rendre probable la formation de créateurs, de techniciens de toutes sortes et autres spécialistes des médias. Plus elle parvient à le faire, meilleure est son aptitude à se reproduire.

35. Mais on comprend bien que, s'il n'y pas de médiatisation de messages sans ressources économiques, l'économie ne suffit pas à une communauté pour produire et diffuser des messages.

36. Certains contenus peuvent être produits à peu de frais, grâce, par exemple, à des technologies comme la caméra numérique et Internet. Mais, symboliquement, il reste que les médias imprimés et les médias électroniques, comme la radio et la télévision, bénéficient d'un ascendant symbolique et pratique qui leur donne une forte influence dans les populations.

37. Une communauté aura tous les médias qu'elle voudra, si elle ne peut générer la production de messages, elle ne peut se reconnaître dans ce qu'elle se diffuse. À l'opposé, elle aura beau favoriser la création, si elle ne peut en diffuser les produits, ils ne peuvent être partagés.

\section{Économie, démographie, culture et médias}

38. Dans la postmodernité, l'existence d'une communauté sociétalement importante suppose une interaction de considérations économiques, démographiques, culturelles et médiatiques.

39. Il n'y a pas de communauté sociétalement importante :

a. qui n'ait pas de culture caractéristique. Pour cette communauté, se reproduire, c'est assurer son être culturel ;

b. qui n'ait pas de démographie significative. Cette démographie permet de caractériser l'ensemble en en spécifiant des traits distinctifs (langue, religion, localité) et, surtout, elle donne à observer un ensemble suffisamment nombreux pour qu'il puisse justifier l'existence des institutions qui sont nécessaires à sa reproduction ;

c. qui n'ait pas de structure économique fonctionnelle. Cette structure est nécessaire à l'entretien et au développement du système institutionnel ; 
d. qui n'ait pas de structure médiatique. C'est largement sur cette structure que repose la possibilité d'assurer la spécificité de l'être communautaire aussi bien dans ses particularismes que dans ses traits collectifs.

40. Mais on le voit bien : on a moins affaire ici à des corrélations qu'à un ensemble intriqué de relations. Si la démographie n'est pas significative, la structure économique en souffre. Sur le plan médiatique, du point de vue du diffuseur, dans une logique marchande, cela veut dire qu'il n'y a pas suffisamment de destinataires pour justifier l'émission de messages, aussi bien leur production que leur transmission. Ainsi, la culture d'une communauté ne trouve pas les médias qui lui permettront de circuler au sein de l'ensemble ou dans un sous-ensemble. Démographie, culture, médias et économie constituent donc un tout aux éléments interreliés. Une communauté démographiquement nombreuse est plus susceptible qu'une autre, moins populeuse, de justifier les investissements privés qui auront pour fin la production et la diffusion de messages pour elle-même. Il est alors plus probable que des membres de la communauté déploient leurs facultés créatrices pour générer des produits culturels. Si au nombre s'ajoute la richesse, alors la communauté favorisera davantage la concurrence des messages médiatiques produits en elle et pour elle. La communauté pourra alors solliciter ce qui lui sera livré sur les plans médiatiques et culturels, et elle pourra aussi bénéficier de contenus qu'elle n'aura pas sollicités puisqu'elle constituera un lieu propice à la création et à la production de messages culturels.

41. Il existe une dynamique importante entre économie, démographie et médias pour la reproduction de la culture. Une culture suppose une démographie, c'est-à-dire un ensemble d'individus assez grand pour la faire exister et dont la composition lui donne ses traits caractéristiques. Cette démographie est de nature à assurer, politiquement et économiquement, une médiatisation et l'information qui circule en elle ; parce qu'il 
en est ainsi, la médiatisation reproduit elle-même la démographie dans ses formes culturelles. Parce qu'une économie médiatique est possible, pour des raisons démographiques, les médias agissent sur la culture qui, elle-même, influe sur les médias (voir la figure 1).

Figure 1

Interrelation de la culture, de la démographie, des médias et de l'économie

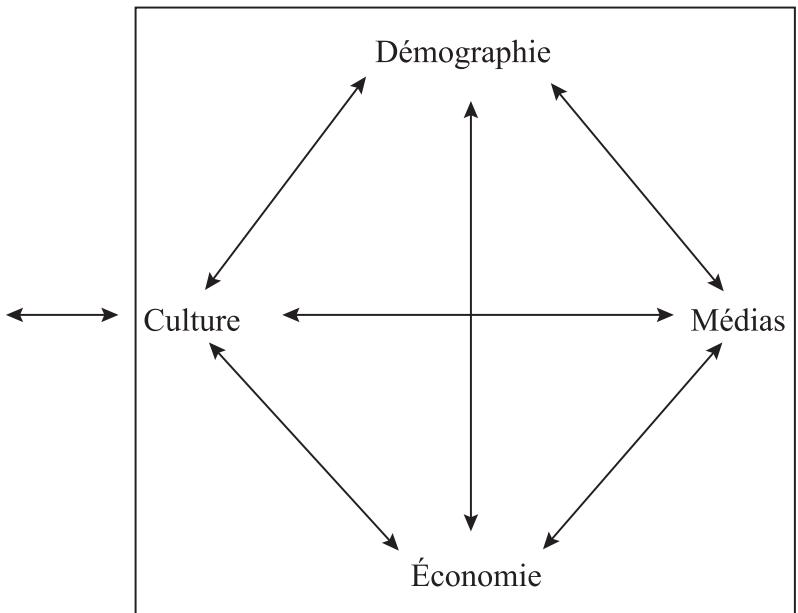

42. La plupart des gouvernements des sociétés postmodernes savent à quel point peut être féconde cette logique économique. C'est pourquoi ils ne tendent pas à lui nuire. En contrepartie, la plupart des États savent à quel point il importe d'intervenir pour minimiser les effets pervers de cette logique dans la mesure où elle est animée à des fins de marchandisation. Car si la production et l'émission de messages médiatiques répondent trop à des intérêts économiques, alors les cultures des communautés risquent d'être menacées. Par exemple, un diffuseur pourrait avoir intérêt à ne pas produire des contenus parce que cela coûte cher, et à les acheter à faible coût chez des producteurs étrangers. Pour cette raison, de nombreux États veillent à ce que même les diffuseurs privés produisent et diffusent des contenus qui permettent aux communautés de se reproduire en recevant 
des messages qui reflètent leur être. Dans le même esprit, si la logique marchande est la seule à s'imposer, il est probable que les communautés minoritaires n'attirent pas les diffuseurs. C'est la raison pour laquelle des gouvernements créent des sociétés d'État qui ont, entre autres, pour mandat de communiquer des messages dans l'intérêt des communautés les moins favorisées. Généralement ces mandats ont à l'esprit les minorités dont l'État a reconnu qu'elles jouissent d'un statut national important et les sociétés publiques de diffusion s'adressent à elles dans une optique généraliste qui permet la reproduction spécifique et collective de la communauté ${ }^{11}$.

\section{L'importance des médias pour les communautés}

43. Les communautés ont besoin de médias pour deux raisons, tout aussi importantes l'une que l'autre. La première a trait à la nécessité du partage de l'information, la seconde au rôle de la concurrence des messages médiatiques.

\section{Les informations partagées et partageables}

44. Toute communauté existe dans la mesure où circulent en elle des informations partagées et partageables. Les informations partagées sont celles qui caractérisent la communauté. Il s'agit de valeurs et de symboliques communes : des principes, des héros, des traditions, des œuvres, des histoires, des thèmes, des manières de faire ou de dire, toutes ces réalités dont est habité l'imaginaire collectif. Les informations partageables sont celles qui relèvent de l'histoire au présent. Il s'agit des événements qui surviennent dans la communauté et de ceux qui ont lieu dans son environnement extérieur. Ce qui arrive en elle doit être communiqué à l'ensemble pour que cela devienne effectivement collectif; et parce que la communauté doit donner un sens à cet événement, qu'elle doit le faire sien, il faut que circulent en elle les diverses interprétations qu'elle

11. Simon Laflamme, «Les Médias en milieu minoritaire : les rapports entre l'économie et la culture », dans Fernand Harvey (dir.), op. cit., p. 25-35. 
peut lui donner. Ce qui se produit en dehors d'elle doit être identifié comme pertinent, diffusé à travers les membres et, ici encore, interprété. Dès lors que ces opérations informationnelles ne peuvent pas avoir lieu dans le seul cadre des échanges in praesentia, l'existence des médias et les qualités du système médiatique déterminent la possibilité même de la communauté.

\section{La concurrence des messages médiatiques}

45. Dans la postmodernité, la concurrence des messages médiatiques est immensément forte. En fait, les sociétés postmodernes sont caractérisées par la concurrence de ces messages, facteur qui contribue à la production de la diversité sociale.

46. Cette concurrence est externe ou interne.

47. Elle est interne quand, au sein d'une même communauté, divers médias s'offrent à la population et que, de surcroît, un même média rend possibles plusieurs expressions. Quand il en est ainsi, cela signifie que la communauté dispose de nombreux diffuseurs qui lui permettent de faire circuler en elle des informations partagées et partageables. En fait, plus est grande la concurrence des messages médiatiques au sein d'une communauté, plus il lui est possible d'assurer sa reproduction. Quand cette concurrence est grande, en effet, la communauté parvient à se situer dans la double tendance à la différenciation et à l'homogénéisation. Il lui est alors facile de donner cours aux diverses expressions qu'elle porte en elle ou qu'elle peut générer en même temps qu'il lui est aisé de diffuser les propos rassembleurs qui font, par exemple, que, même dans le débat, les citoyens se retrouvent. Les sociétés les plus vigoureuses, dans la postmodernité, ne sont pas celles dans lesquelles tout le monde dit la même chose sur tout; ce sont celles qui peuvent débattre de tout sur un fonds symbolique partagé, qui, donc, ont un fonds médiatique concurrentiel important. La concurrence médiatique, au demeurant, est d'autant plus forte que la communauté est nombreuse et riche, que, donc, 
elle dispose du système institutionnel qui rend possible la vie en fonction des caractéristiques de la communauté. Si tel est le cas, par exemple, la communauté dispose des écoles qui favorisent la créativité et des infrastructures qui favorisent la production et la diffusion de messages.

48. La concurrence est externe quand des messages qui circulent dans une communauté ne lui proviennent pas de ses propres diffuseurs ou qu'ils n'ont pas été produits par elle. Si la concurrence interne est forte, ces messages externes ont généralement peu de conséquences en ce sens qu'ils ne menacent pas la reproduction de la communauté. Si elle est faible, la communauté est vulnérable.

49. Les messages peuvent être externes à divers niveaux. Cela dépend principalement de l'éloignement de leurs caractéristiques par rapport aux éléments qui définissent la communauté qui les reçoit. À l'extrême, le message qui est communiqué à une communauté n'a pas été produit par elle, n'est pas interprété par le diffuseur, ne reflète pas ses modes de vie, ne correspond pas à ses us culturels et artistiques, lui est transmis dans une langue autre que celle qui est parlée par les membres. Si tel est le cas, il est fort probable qu'il remue la communauté, et il l'est d'autant plus que, si ce contenu lui parvient, c'est qu'il est enveloppé d'une impressionnante aura, qu'il est précédé d'une puissante charge symbolique. À un moindre degré, le contenu externe a de fortes ressemblances avec les contenus qui circulent dans la communauté : us et coutumes comparables, langue identique, modes artistiques et culturels similaires. Dans ces conditions, la communauté arrive à reproduire une partie d'elle-même. Mais dans un cas comme dans l'autre, il y a une distance entre le message diffusé et les particularités de la communauté ; et les effets de cette distance sont d'autant plus menaçants que la part de ces messages externes est grande dans l'ensemble des messages diffusés. Même si ces messages ont une forte proximité avec l'imaginaire collectif de la communauté qui les reçoit, s'ils 
sont trop nombreux et qu'ils ne parviennent pas à interpeller la communauté pour elle-même, dans son vécu spécifique, il est fort probable que des membres délaissent ces messages pour se tourner vers ceux qui sont symboliquement valorisés à l'extérieur d'elle-même. Car à quoi lui servirait-il de s'exposer de façon soutenue à des messages qui s'adresseraient à elle, mais qui ne pourraient assurer l'évolution de son être spécifique dans la vie de tous les jours!

50. Dans une société de concurrence des médias, si une communauté ne trouve pas dans ce qui, théoriquement, s'adresse à elle, ce qui lui est utile et qui est malgré tout diffusé dans le champ médiatique, alors elle tendra à se tourner vers ce qu'offre le champ médiatique et ce qui est perçu comme pratique. Car, dans ces autres médias, il y aura aussi des correspondances avec son éthos. Et plus il y a de proximité entre les autres médias qui ne s'adressent pas à elle en tant que communauté et les caractéristiques de la communauté, plus il est probable que la communauté se détourne des messages qui s'adressent à elle mais ne la ramènent pas à son vécu.

51. Si la communauté est une minorité et qu'elle est peu desservie par ses médias, il est fort probable qu'elle se tourne vers les médias de la majorité. Car, généralement, elle découvrira là des contenus auxquels elle pourra se rattacher soit parce qu'ils correspondent à quelques aspects de ses symboliques, soit parce qu'ils comportent des messages utiles à la vie quotidienne. Et le média de la quotidienneté par excellence, c'est la radio. Il est rare qu'une communauté minoritaire ne se reconnaisse aucunement dans le discours d'une communauté majoritaire. La postmodernité, par la concurrence des médias et des messages médiatiques qu'elle implique, rend peu probable, sur le plan symbolique, une grande distance entre les contenus diffusés et les traits d'une communauté. Cela a pour effet que le minoritaire peut aisément se tourner vers les médias du majoritaire s'il ne dispose pas de médias pour lui-même ou si ces médias ne correspondent pas à ses 
attentes. Ce faisant, il en vient à se reproduire au rythme de ce qui est véhiculé dans les médias qui ne proviennent pas de sa communauté. La fragile distance qu'il y a entre les traits de sa communauté et les caractéristiques des messages extérieurs qu'il reçoit s'estompe et l'on assiste à un glissement vers l'éthos de la majorité.

\section{Des médias pour la communauté}

52. Dans la postmodernité, une communauté sociétalement importante ne peut se reproduire que si des messages sont produits par elle-même et pour elle-même. Si tel n'est pas le cas, la communauté se tourne vers les messages qui ne sont produits ni par elle ni pour elle. Ainsi, elle se vulnérabilise.

53. Une communauté qui est en mesure de produire des contenus informationnels et divertissants et qui, de surcroît, se donne les moyens de se les diffuser se donne simultanément les moyens de sa reproduction, car il lui est alors possible de se reconnaître à travers les messages qu'elle reçoit et de partager des symboliques. Et cette reproduction est d'autant plus probable que les contenus qui relèvent de sa propre socialité occupent un espace important dans l'ensemble des messages qui sont diffusés.

54. Cet effet des messages qui sont issus de la communauté est d'autant plus bénéfique qu'ils sont repris par d'autres communautés, à l'intérieur de la société et même à l'extérieur. Car revient alors à la communauté une image d'elle-même qui plaît ailleurs, ce qui est l'une des manifestations les plus valorisantes pour une communauté spécifique. Le minoritaire qui voit qu'on apprécie dans la majorité, et même au-delà, ce qui provient de sa communauté tend à trouver là des motifs de valorisation.

55. Rares sont les communautés, dans les sociétés postmodernes, qui n'ont pas quelque chose en partage avec des populations qui vivent ailleurs, dans d'autres sociétés. Il y a là un facteur important de renforcement culturel. Une minorité linguis- 
tique, par exemple, dont la langue est importante en dehors de la société, donnant cours, par exemple, à une forte production médiatique ailleurs, et donc dans le monde, peut alimenter ses médias de cette production externe et en faire des sources de valorisation intérieure. Mais cette possibilité d'alimenter le paysage médiatique interne par les productions externes est d'autant plus utile à la communauté que ces productions ne se substituent pas à la production interne de contenus ${ }^{12}$.

\section{Ontario Français ET Francophonie MinORITAIRE AU CANADA Une minorité importante dans des situations différentes}

56. La communauté franco-ontarienne est minoritaire. Elle l'est par référence à un ensemble qu'on pourrait définir comme essentiellement anglo-ontarien. Sa position de minoritaire est avant tout affaire de démographie.

57. Au recensement de 2001, on a posé la question suivante : «Quelle est la langue apprise en premier lieu à la maison dans l'enfance et encore comprise? ». Si la personne a choisi « français » comme réponse unique ou comme composant d'une réponse multiple, alors la proportion de francophones de l'Ontario est de 4,8\%, soit 548940 individus sur 11410045 Ontariens ( si l'on ne retenait que les réponses uniques, cette proportion serait de $4,3 \%)^{13}$.

12. Simon Laflamme, « Les Médias en milieu minoritaire : les rapports entre l'économie et la culture », op. cit., ; Armand Mattelart et Jean-Marie Piemme, Télévision, enjeux sans frontières : industries culturelles et politiques de la communication, Grenoble, Presses universitaires de Grenoble, 1980 ; Bernard Miège, La Société conquise par la communication, Grenoble, Presses universitaires de Grenoble, 1989.

13. Ce critère a été délaissé depuis : «Pour des raisons historiques, Statistique Canada a généralement utilisé le critère de la langue maternelle, soit la première langue apprise à la maison dans l'enfance et encore comprise au moment du recensement. Les statistiques fondées sur la langue maternelle ont l'avantage d'être approximativement comparables depuis plus d'un demi-siècle » (Statistique Canada,http://www.statcan. gc.ca/pub/89-642-x/2010001/article/section1-fra.htm, site consulté en mars 2018).

« Le gouvernement de l'Ontario a adopté, le 4 juin 2009, la Définition inclusive de francophone (DIF). Cette définition est fondée sur trois questions du recensement au sujet de la langue maternelle, de la langue parlée à la maison et de la connaissance des langues officielles. Antérieurement, la définition identifiait les francophones en vertu du français comme langue apprise à la maison et encore comprise lors du recensement. 
58. Mais les Franco-Ontariens ne sont pas partout pareillement minoritaires. La communauté dans son ensemble connaît des variations importantes selon les régions, en termes absolus et relatifs (voir le tableau 1). Les francophones sont plus nombreux dans l'Est (226 705) ; dans le Nord-Est, ils représentent la minorité proportionnellement la plus grande $(25,1 \%)$. Dans le Nord-Ouest, leur ensemble est le plus petit, en chiffres absolus (8 780) et relatifs (3,7\%). Dans le SudOuest, ils comptent pour 6,3\% de tous les Franco-Ontariens de la province et pour $2,3 \%$ de la population de la région. On le voit : on a bien affaire à une minorité importante dont la situation démographique n'est cependant pas partout la même.

\section{Une minorité qui se compare à la majorité}

59. Le fait de la minoritude démographique ne correspond pas à une inégalité universelle : en comparant la communauté franco-ontarienne à la totalité des Ontariens, on n'a jamais l'évidence d'une nette discrimination, quoiqu'on relève des signes de minorisation. En se référant aux données du recensement de 2001, l'Office des affaires francophones signale, en effet, que :

a. la proportion de francophones (11,9\%) qui n'a pas atteint la $9^{\mathrm{e}}$ année de scolarité est plus élevée que celle de la population générale $(8,1 \%)$;

b. la proportion de francophones $(67,0 \%)$ qui détient un diplôme d'études secondaires est plus faible que celle de la population générale $(70,3 \%)$;

c. la proportion de francophones $(77,9 \%)$ qui a fait des

« L'Ontario a connu une augmentation de la population francophone de 28805 personnes entre 2006 et 2011 pour un total de 611 500, soit 4,8\% de la population totale de la province

[...]

« En 2011, 525960 personnes vivant en Ontario ont déclaré avoir le français comme langue maternelle, contre 510240 en 2006. En 2001, ces personnes représentaient 4,1\% de la population, contre 4,2\% en 2006 » (Gouvernement de l'Ontario, Ministère des finances, http://www.fin.gov.on.ca/fr/economy/demographics/census/cenhi11-6.html, site consulté en mars 2018). 


\section{Tableau 1}

Distribution de la population francophone de l'Ontario selon la région

\begin{tabular}{|l|r|r|r|r|r|}
\hline \multicolumn{5}{|c|}{ (Réponses multiples à la question du recensement de 2001 : } \\
" Quelle est la langue apprise en premier lieu à la maison dans l'enfance \\
et encore comprise ? »)
\end{tabular}

études postsecondaires est plus faible que celle de la population générale $(79,5 \%)$;

d. dans l'ensemble des personnes qui ont fait des études postsecondaires, la proportion de francophones $(45,0 \%)$ qui ont étudié à l'université est plus faible que celle de la population générale $(49,0 \%)$;

e. la proportion de francophones $(14,7 \%)$ qui ont obtenu un diplôme d'études universitaires est plus faible que celle de la population générale $(17,5 \%)$;

f. la proportion de francophones (59,2\%) actifs sur le marché du travail est plus faible que celle de la population générale $(61,5 \%)$;

g. le taux de chômage des hommes francophones $(6,5 \%)$ est plus élevé que celui de l'ensemble masculin (5,8\%), mais le taux de chômage des femmes francophones $(5,7 \%)$ est inférieur à celui de la population féminine (6,5\%); 
h. la structure professionnelle de la population francophone est relativement semblable à celle de l'ensemble de la population générale ; on note toutefois une surreprésentation de francophones dans le domaine de l'enseignement;

i. le revenu médian des francophones (30 054 \$) est supérieur à celui de la population générale (28 027 \$);

j. le revenu d'emploi moyen des francophones $(35796 \$$ ) dépasse légèrement celui de la population générale (35 185 \$);

k. le revenu familial moyen des francophones (70 $033 \$$ ) est inférieur à celui de la population dans son ensemble (73 $849 \$) .{ }^{14}$

60. Si, donc, la population franco-ontarienne est démographiquement inférieure, elle ne représente pas un ensemble ségrégué. Si l'on se fie à des indicateurs socio-économiques comme l'instruction, l'emploi et le revenu, on est forcé de constater que sa situation se compare assez bien à celle de la majorité - il n'y a pas, en effet, pour les inégalités observées, d'écarts incommensurables - ; cela signifie que les francophones, malgré leur minorisation, savent tirer profit des institutions dont ils disposent.

\section{Une minorité qui dispose de la même technologie médiatique}

61. Si l'on compare les Franco-Ontariens aux Anglo-Ontariens quant à la technologie médiatique dont ils disposent dans leurs foyers, on découvre que les deux populations sont très semblables. En effet, une enquête révèle qu'il n'y a pas de différences de proportions notables entre les deux groupes linguistiques pour ce qui est des principales technologies médiatiques dont on peut faire usage ou des services médiatiques auxquels on peut recourir : téléviseur, appareils radio,

14. Office des affaires francophones en Ontario, Les Francophones en Ontario. Profil statistique. Profil général, Toronto, Gouvernement de l'Ontario, 2005 ; Simon Laflamme et Derek Wilkinson, « Portraits à grands traits de l'Ontario français », Québec Studies, vol. 46, Fall 2008 / Winter 2009, p. 7-18. 
télécopieur, branchement Internet, lecteur DvD, service du câble, antenne parabolique, ligne téléphonique, téléphone cellulaire.

Cela signifie que les francophones de la province sont en mesure de capter les mêmes messages que les autres Ontariens. Par conséquent, ils sont à même de s'exposer aux messages médiatiques qui ne s'adressent pas à eux de façon spécifique, ils ont les moyens de se tourner vers les messages qui s'adressent à la majorité. Et ils le font ${ }^{15}$.

\section{Une puissante anglicité}

62. On ne peut pas dire que les Franco-Ontariens soient socioéconomiquement en position d'infériorisation. On ne peut pas dire que leurs foyers témoignent d'une marginalisation médiatique. Mais on ne peut pas affirmer non plus que leur position sociétale soit assurée ; on ne peut surtout pas déclarer que leurs comportements médiatiques soient le signe d'une reconnaissance de soi.

63. Les Franco-Ontariens - tout comme les Acadiens - disposent d'un système institutionnel plutôt élaboré, quoique grandement perfectible et dont les qualités ne sont pas partout les mêmes sur le territoire de la province. Cela contribue grandement à leur émancipation, dans l'ensemble. Leur province - tout comme le Nouveau-Brunswick - est à proximité du Québec, un territoire à majorité francophone. Cette contiguiité leur est, à certains égards, bénéfique : elle favorise les liens personnels ou institutionnels de chaque côté de la frontière - notamment dans l'Outaouais - ; elle permet à la minorité ontarienne d'importer des messages médiatiques avec lesquels elle aura une certaine affinité ; elle permet aux créateurs franco-ontariens de se faire connaître ailleurs qu'en Ontario sur le territoire national, ce qui valorise la conscience franco-ontarienne. Mais les Franco-Ontariens, tout comme les

15. Simon Laflamme, « Les Médias en Ontario chez les francophones et les anglophones », Cahiers Charlevoix, Sudbury, Société Charlevoix / Prise de parole, $\mathrm{n}^{\circ} 6$, 2004, p. 251-282. 
autres francophones du Canada hors Québec, vivent au sein de la majorité qui est la plus grande productrice de messages médiatiques de la planète : le monde anglophone. Le Canada anglais est un fort producteur de messages médiatiques et le Canada a une longue frontière avec le producteur de messages médiatiques le plus puissant. En outre, le monde anglophone ailleurs produit de nombreux contenus médiatiques qui sont aisément importés au Canada. La francophonie, certes, occupe un espace important dans l'ensemble des messages qui sont produits sur la planète, mais cet espace est loin de pouvoir rivaliser avec celui de l'anglophonie. Cette situation rend difficile la valorisation de soi du Franco-Ontarien; elle la rend telle d'autant plus que ce minoritaire parle normalement la langue de la majorité, ce qui élimine la barrière linguistique.

64. Cette puissance anglophone se révèle dans l'imaginaire collectif. Nombre de jeunes déprécient leur francité, sont interpellés par l'anglicité. Des adultes, surtout parmi les moins instruits, sont dans le même état d'esprit ${ }^{16}$.

\section{Entre le français et l'anglais sur Internet}

65. La résistance au français et l'attrait de l'anglais se répercutent dans les comportements médiatiques.

66. Dans le nord-est de l'Ontario, on a interrogé des jeunes sur la langue d'usage dans la navigation sur Internet; on leur a demandé d'estimer la proportion du temps où ils naviguaient en français. La réponse moyenne est de $32 \%$. Cela signifie donc que les jeunes francophones de l'Ontario emploient le français de façon relativement importante quand ils circulent sur le Web ; cela veut également dire que cette circulation a

16. Simon Laflamme et Donald Dennie, L'Ambition démesurée. Enquête sur les aspirations et les représentations des étudiants et des étudiantes francophones $d u$ Nord-Est de l'Ontario, Sudbury, Prise de Parole/Institut franco-ontarien, 1990. Simon Laflamme et Pierre Bouchard, Les Jeunes et le Nord. Un parcours à découvrir, Hearst, Commission de formation du Nord-Est, 2005. Avec Pierre Bouchard, http://simonlaflamme.ca/wp/wp-content/uploads/2016/07/LesjeunesetleNordabregefinal-2005. pdf; Simon Laflamme, « Passage de l'élémentaire au secondaire et décrochage culturel en Ontario français. Élimination de quelques facteurs », Cahiers Charlevoix. Études franco-ontariennes, Ottawa, Presses de l'Université d'Ottawa, no 9, 2013, p. 15-59. 
principalement lieu en anglais, soit le temps restant : $68 \%$ (la part des langues autres que le français et l'anglais est à peu près nulle). Il faut toutefois attirer l'attention sur un phénomène important : les différences entre les individus. L'écart-type de cette moyenne de $32 \%$ est de $33 \%$. Cela implique qu'il y a énormément de variations entre les individus, que certains emploient peu le français, mais que d'autres y recourent abondamment.

Ces chiffres rappellent à quel point la situation des francophones de l'Ontario représente un vaste champ de possibilités. S'il y a des mouvements de nette assimilation, il y a aussi d'importantes affirmations culturelles. ${ }^{17}$

\section{Minorités linguistiques au Québec et ailleurs au Canada}

67. C'est en comparant la minorité anglophone du Québec aux minorités francophones du Canada qu'on mesure le mieux la force attractive de l'anglais dans les médias. Pour ce faire, on peut utiliser les données de l'Enquête sur la vitalité des minorités de langue officielle (2006) (ne sont ici pris en considération que les adultes de 15 ans et plus).

68. On peut procéder à la comparaison en se donnant une échelle de 1 à 5 où la valeur de « 1 » signifie « seulement la langue de la majorité » et celle de « 5 », « seulement la langue de la minorité ». Au Québec, donc, « 5 » signifie « seulement l'anglais » et, ailleurs au Canada, « seulement le français ». Ainsi, plus la moyenne est élevée, ou proche de 5 , plus la population tend à s'exposer aux médias dans sa langue. On peut accoler cette échelle à des médias éminemment importants comme Internet, la télévision, la radio, les journaux et les livres.

69. L'analyse révèle que les anglophones du Québec tendent beaucoup plus que les francophones des autres provinces à opter pour leur langue maternelle quand ils s'exposent à un

17. Simon Laflamme, «Usage et représentation d'Internet chez les jeunes. Comparaison entre les francophones et les anglophones du nord de l'Ontario ", Cahiers Charlevoix, Sudbury, Société Charlevoix / Prise de parole, $n^{\circ} 7,2006$, p. $183-$ 228. 
média (voir le tableau 2). Les chiffres sont éloquents : ils sont presque tous supérieurs à 4 pour les Anglo-Québécois ; ils franchissent difficilement la valeur de 2 pour les minorités francophones. L'anglo-minoritaire s'oriente fortement vers les médias qui parlent sa langue, le franco-minoritaire le fait beaucoup moins volontiers. Certes, l'accessibilité des médias a ici quelque incidence sur ces statistiques, surtout qu'on est en situation de concurrence des messages médiatiques. Mais il faut constater qu'on a aussi affaire à un phénomène fortement symbolique, ce que démontre sans l'ombre d'un doute le rapport à Internet puisqu' on peut aisément circuler sur le Web en français et que les francophones hors Québec le font avec une évidente modération. Les chiffres montrent également que c'est la radio qui incite le plus les franco-minoritaires à recourir au français. L'exposition à la radio étant davantage affaire de disponibilité que la navigation sur Internet, on peut aisément comprendre que, si s'ajoute à l'avantage symbolique de l'anglais l'indisponibilité du média ou de contenus radiophoniques pertinents pour le francophone, alors la probabilité qu'on l'écoute en français est très faible.

70. Une nouvelle fois, il ne faut pas négliger les écarts-types. Ces mesures de dispersion soulignent le fait que, dans les deux contextes, il y a de grandes différences entre les individus. Ces statistiques tournent autour de la valeur de 1, sur une échelle à 5 niveaux; cela est loin d'être négligeable. Ainsi, si les tendances centrales montrent qu'il est clair que les AngloQuébécois, dans l'ensemble, et les franco-minoritaires, dans l'ensemble également, se comportent de façon divergente pour ce qui est de la langue d'usage de leurs médias, les mesures de dispersion, elles, rappellent que, dans les deux populations, les individus n'ont pas tous la même attitude. Cela démontre bien, une nouvelle fois, que si l'orientation vers l'anglais est partout manifeste, elle est loin d'être absolue et que, par conséquent, en intervenant sur les infrastructures 
Tableau 2

Moyennes et écarts-types de la langue d'usage pour divers médias selon la région où se situe la minorité linguistique

\begin{tabular}{|l|c|c|}
\hline \multicolumn{3}{|c|}{$\begin{array}{c}\text { Analyse de variance pondérée }\left(\text { en } \mathrm{F}_{\text {Wald }}\right) . \\
\text { Toutes les différences sont inférables à p }<0,001 \\
(1=\text { seulement dans la langue de la majorité } ; \\
5=\text { seulement dans la langue de la minorité })\end{array}$} \\
\hline Média & \multicolumn{2}{|c|}{ Région } \\
\cline { 2 - 3 } & Extérieur du Québec & Québec \\
\hline Internet & $1,99(1,04)$ & $4,41(0,91)$ \\
\hline Télévision & $2,33(1,11)$ & $4,22(0,85)$ \\
\hline Radio & $2,54(1,40)$ & $4,14(1,21)$ \\
\hline Journaux & $2,05(1,40)$ & $3,71(1,46)$ \\
\hline Livres & $2,35(1,30)$ & $4,26(1,15)$ \\
\hline
\end{tabular}

médiatiques, on agit nécessairement sur les destinataires des messages.

\section{Instruction et rapport linguistique aux médias}

71. Pour poursuivre sur ces données, il est opportun de rappeler que le rapport linguistique aux médias n'a pas lieu en vase clos, qu'il dépend, par exemple, du niveau d'instruction et, plus encore, de la langue dans laquelle les études ont eu lieu.

72. On note, par exemple, que les minoritaires qui ont fait des études universitaires tendent plus que ceux qui ne se sont pas instruits au-delà du secondaire à s'exposer au français.

73. On constate également que le fait d'avoir étudié en français, pour le francophone minoritaire, accroît la probabilité qu'il choisisse sa langue quand il recourt aux médias, et ce, pour tous les niveaux d'instruction. Si l'on prend, au Canada français hors Québec, les personnes qui n'ont pas fait d'études postsecondaires et qu'on les divise selon que les études qui ont été faites l'ont été en français ou non, on remarque que les moyennes sont toujours plus élevées pour la première population (voir le tableau 3). Cela signifie que, pour le 
franco-minoritaire, le fait de s'être scolarisé dans sa langue favorise, dans l'exposition aux médias, l'usage du français, même s'il n'a fait aucune étude postsecondaire.

\section{Tableau 3}

Moyennes (écarts-types) de la langue d'usage de divers médias quand la personne n'a pas fait d'études postsecondaires et que ses études ont été faites ou non en français

\begin{tabular}{|l|c|c|}
\hline \multicolumn{4}{|c|}{$\begin{array}{c}\text { Analyse de variance pondérée }\left(\text { en } \mathrm{F}_{\text {Wald }}\right) \\
\text { Toutes les différences sont inférables à p }<0,001 \\
\text { (Pour l'usage des médias : } 1=\text { seulement l'anglais } \\
\text { et 5 = seulement le français) }\end{array}$} \\
\hline Média & $\begin{array}{c}\text { A fait ses études } \\
\text { en français }\end{array}$ & $\begin{array}{c}\text { N'a pas fait ses études } \\
\text { en français }\end{array}$ \\
\hline Internet & $2,38(0,99)$ & $1,86(1,02)$ \\
\hline Télévision & $2,53(1,03)$ & $2,16(1,12)$ \\
\hline Radio & $2,71(1,42)$ & $2,14(1,38)$ \\
\hline Journaux & $2,36(1,38)$ & $1,97(1,39)$ \\
\hline Livres & $2,83(1,15)$ & $2,22(1,31)$ \\
\hline
\end{tabular}

74. Si l'on procède à la même comparaison, mais pour l'ensemble des individus qui ont fait des études collégiales, la conclusion est la même : s'instruire dans sa langue, pour le francominoritaire, c'est augmenter la probabilité d'employer le français dans les médias (voir le tableau 4).

75. $\mathrm{Si}$, enfin, on ne retient que les personnes qui ont fait des études universitaires, la tendance est tout à fait corroborée (voir le tableau 5).

76. Ainsi, il y a une nette corrélation entre la langue de l'instruction et la langue d'exposition aux médias. Ces observations rappellent à quel point les institutions sont liées entre elles dans l'organisation sociale d'une communauté. Elles démontrent que, s'il est vrai que l'anglais est interpellant pour le franco-minoritaire, il n'est pas moins vrai que, en consolidant la structure institutionnelle de sa communauté, on 


\section{Tableau 4}

Moyennes (écarts-types) de la langue d'usage de divers médias selon que les études qui mènent au diplôme collégial ont été faites ou non en français

\begin{tabular}{|l|c|c|}
\hline \multicolumn{3}{|c|}{$\begin{array}{c}\text { Analyse de variance pondérée }\left(\text { en } \mathrm{F}_{\text {Wald }}\right) \\
\text { Toutes les différences sont inférables à } \mathrm{p}<0,001 \\
\text { (Pour l'usage des médias : } 1=\text { seulement l'anglais } \\
\text { et } 5=\text { seulement le français) }\end{array}$} \\
\hline Média & $\begin{array}{c}\text { A fait ses études } \\
\text { en français }\end{array}$ & $\begin{array}{c}\text { N'a pas fait ses études } \\
\text { en français }\end{array}$ \\
\hline Internet & $2,27(1,11)$ & $1,96(1,02)$ \\
\hline Télévision & $2,47(1,02)$ & $2,20(1,12)$ \\
\hline Radio & $2,49(1,37)$ & $2,23(1,41)$ \\
\hline Journaux & $2,45(1,47)$ & $2,30(1,30)$ \\
\hline Livres & $2,81(1,27)$ & $2,21(1,20)$ \\
\hline
\end{tabular}

\section{Tableau 5}

Moyennes (écarts-types) de la langue d'usage de divers médias selon que les études qui mènent au diplôme universitaire ont été faites ou non en français

\begin{tabular}{|l|c|c|}
\hline & \multicolumn{2}{c|}{$\begin{array}{c}\text { Analyse de variance pondérée }\left(\text { en } \mathrm{F}_{\text {Wald }}\right) \\
\text { Toutes les différences sont inférables à p }<0,001 \\
\text { (Pour l'usage des médias : } 1=\text { seulement l'anglais } \\
\text { et 5 = seulement le français) }\end{array}$} \\
\hline Média & $\begin{array}{c}\text { A fait ses études } \\
\text { en français }\end{array}$ & $\begin{array}{c}\text { N'a pas fait ses études } \\
\text { en français }\end{array}$ \\
\hline Internet & $2,19(1,19)$ & $1,92(0,97)$ \\
\hline Télévision & $2,51(1,24)$ & $2,07(1,00)$ \\
\hline Radio & $2,51(1,53)$ & $2,12(1,31)$ \\
\hline Journaux & $2,40(1,60)$ & $1,86(1,24)$ \\
\hline Livres & $2,68(1,47)$ & $2,21(1,20)$ \\
\hline
\end{tabular}

favorise également les comportements qui sont l'expression de son appartenance communautaire ${ }^{18}$.

18. Une partie de ces analyses a été présentée au Colloque international Langue et territoire, Université Laurentienne, Sudbury, 29 août-3 septembre 2010. Cette communication sera ensuite publiée dans les actes du colloque : Christiane Bernier, Simon 


\section{LES FRANCOPHONES DU SUD-OUEST DE L'ONTARIO}

77. Les francophones du sud-ouest de l'Ontario représentent un groupement particulier de la communauté franco-ontarienne. Ce qui vaut pour l'ensemble, à maints égards, vaut aussi pour eux. Mais ils sont, par certains points, plus vulnérables que d'autres. Leur nombre relativement faible, leur proportion relativement petite au sein de leur région, leur proximité par rapport au territoire étatsunien sont largement responsables de leur vulnérabilité ${ }^{19}$.

78. Quand on compare leurs usages linguistiques des médias à ceux des francophones des régions du Nord ou de l'Est, on se rend compte que leur inclination vers le français est parfois un peu moins prononcée qu'elle ne l'est pour les autres, mais tout particulièrement chez les adolescents.

79. Dans une enquête, on a demandé à des francophones de divers milieux en Ontario d'indiquer selon quelle fréquence ils s'exposaient en français à divers médias. Une valeur de « 1 » correspond à « jamais »; une valeur de « 4 », veut dire « très souvent ».

Laflamme et Sylvie Lafrenière, « Exposition aux médias en milieu minoritaire au Canada. Une comparaison Franco-Ontariens/Anglo-Québécois », dans Ali Reguigui et Julie Boissonneault (dir.), Langue et territoire. Études en aménagement linguistique / Language and Territory: Studies in Language Planning, Sudbury, Université Laurentienne, "Série monographique en sciences humaines / Human Sciences Monographic Series », vol. 14, 2014, p. 195-222. On trouvera les autres analyses et plusieurs raffinements dans Christiane Bernier, Simon Laflamme et Sylvie Lafrenière : « Dans quelle langue officielle s'expose-t-on aux médias en milieu canadien », dans Rodrigue Landry (dir.), La Vie dans une langue officielle minoritaire au Canada, Québec, Presses de l'Université Laval, "Langues officielles et sociétés ", 2014, p. 173-194 ; «L'effet de la disponibilité des médias et de la densité de la population minoritaire sur la langue d'exposition aux médias », Minorités linguistiques et société, $\mathrm{n}^{\circ} 3,2013$, p. 120-143 ; " Dissociation entre perceptions et pratiques. De la langue d'exposition aux médias en milieu minoritaire canadien », Revue du NouvelOntario, no 39, 2014, p. 83-163.

19. On lirait avec intérêt sur cette question le livre d'Anne Gilbert, Espaces franco-ontariens. Essai, Ottawa, Le Nordir, 1999, et les textes d'Yves Frenette, "L'Ontario français du Centre et du Sud-Ouest, 1940-1970 », Cahiers Charlevoix : études franco-ontariennes, $\mathrm{n}^{\circ}$ 7, 2006, p. 145-183 et " Aspects de 1'histoire des Franco-Ontariens du Centre et du Sud-Ouest, 1970-2000 », Cahiers Charlevoix : études franco-ontariennes, $\mathrm{n}^{\circ}$ 10, Ottawa, Presses de l'Université d'Ottawa, 2014, p. 211-254. 
80. Si l'on prend la télévision, on note que, parmi les jeunes qui ont 13 ans ou moins, dans le Sud-Ouest, la moyenne est de 1,55, qu'elle est un peu plus faible dans le Centre $(1,27)$, mais qu'elle est plus élevée dans les trois autres régions $(2,21$ à 2,44) (voir la figure 2). Chez les adolescents, le schème est à peu près le même : la moyenne est plus faible dans le Sud-Ouest et dans le Centre $(1,27$ et 1,33) et plus élevée ailleurs : de 1,59 à 2,17. Chez les adolescents du Sud-Ouest et du Centre, l'exposition au français télévisuel est pour ainsi dire nulle. Chez les personnes qui ont entre 19 et 44 ans, la moyenne de l'échantillon du Sud-Ouest est la plus faible, à 2,29 ; mais elle est assez proche des moyennes des autres régions (2,33 à 2,52). Chez les plus vieux, la moyenne dans le Sud-Ouest $(2,84)$ est supérieure à celles qu'on observe dans le Centre (2,37) et dans l'Est $(2,83)$, mais elle est inférieure à celle qu'on trouve dans le Nord-Ouest $(3,09)$ et dans le Nord-Est (3,57). Ainsi, chez les adultes, même dans le SudOuest et le Centre, regarder la télévision en français n'est pas inhabituel.

81. Si l'on se penche sur le cas de la radio, on remarque que, chez les plus jeunes, dans le Sud-Ouest $(1,74)$ et le Centre $(1,73)$, les moyennes sont un peu plus faibles qu'ailleurs $(1,80$ à 2,02$)$ (voir la figure 3). Parmi les adolescents, ceux qui habitent le Sud-Ouest présentent la moyenne la plus petite $(1,60)$, mais cette statistique est assez proche de celles des adolescents qui habitent ailleurs (de 1,76 à 1,83). Chez les francophones qui ont entre 19 et 44 ans, il n'y a pas de réelle différence entre les régions, les moyennes oscillant entre 2,25 et 2,36 - cette moyenne-ci étant celle du Sud-Ouest. Parmi les plus vieux, la moyenne de ceux qui résident dans le Sud-Ouest $(2,72)$ se situe entre les moyennes les plus élevées, celles des citoyens du Nord-Ouest et du Nord-Est (3,08 dans les deux cas), et les plus faibles, celles des habitants du Centre $(2,61)$ et de l'Est $(2,52)^{20}$.

20. Simon Laflamme et Christiane Bernier, Vivre dans l'alternance linguistique. 
Figure 2

Regarder la télévision en français selon l'âge et la région

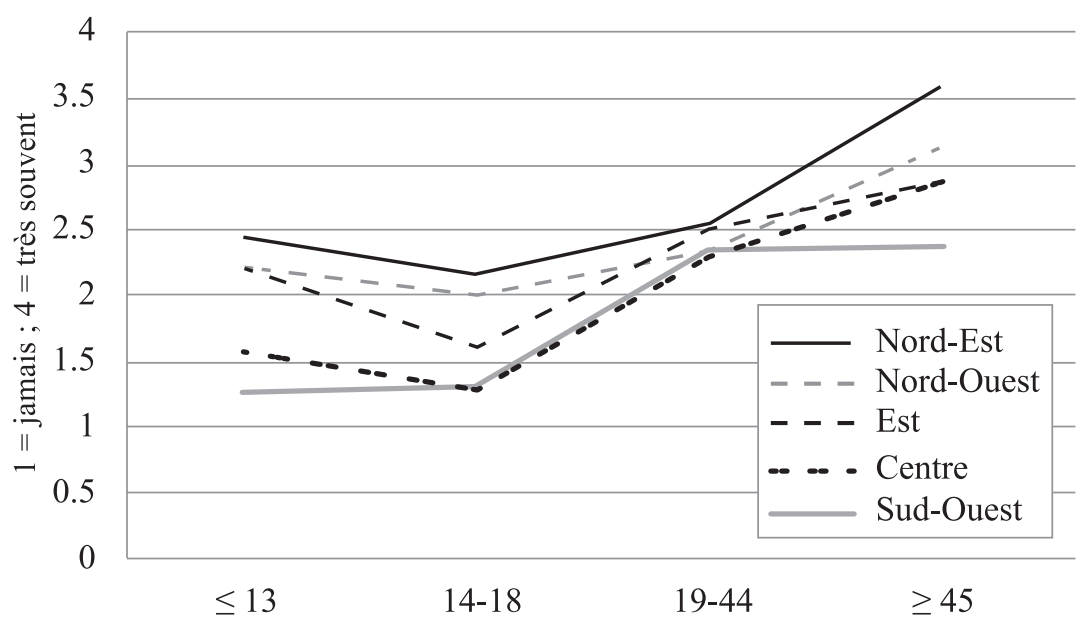

Figure 3

Écouter la radio en français selon l'âge et la région

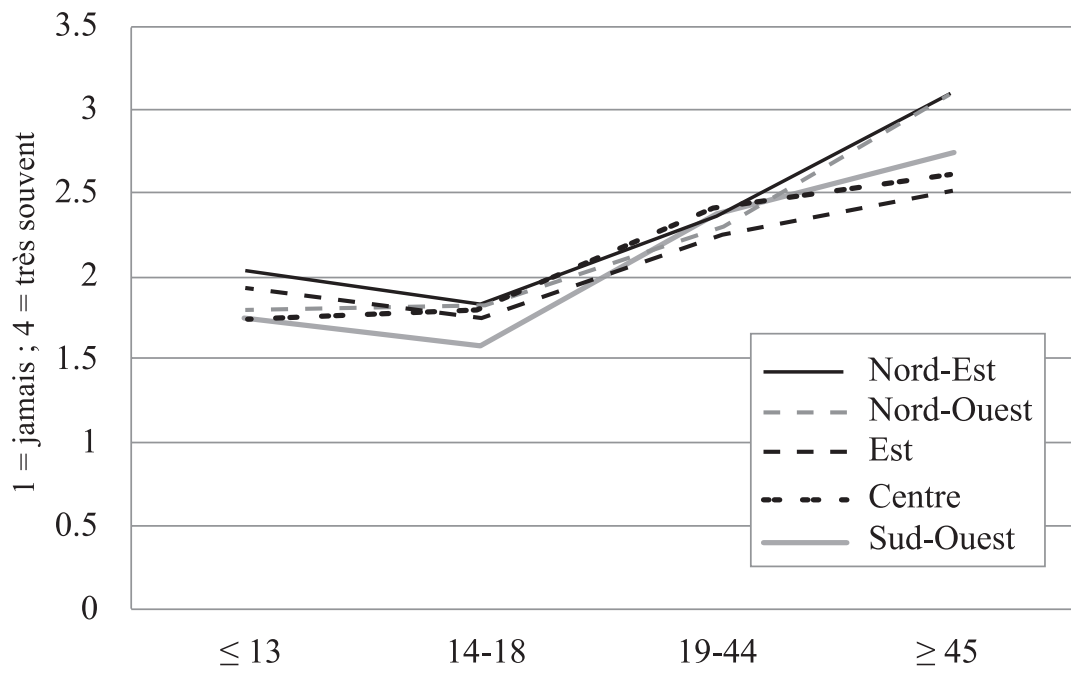


82. Les adolescents sont partout les plus réfractaires à leur langue maternelle, mais cette résistance est plus marquée dans le Centre (pour la télévision) et le Sud-Ouest (pour la télévision et la radio). Parmi les adultes, dans le cas de la télévision, le Sud-Ouest et le Centre inclinent moins vers le français que ne le font les autres régions ; mais telle n'est pas la situation en ce qui concerne la radio. Chez les adultes, le recours au français n'est exceptionnel ni quand on regarde la télévision, ni quand on écoute la radio, et ce, dans aucune des régions. Les adultes du Sud-Ouest écoutent autant la radio en français que ceux des autres régions. Certes, l'anglais interpelle partout, et à tous les âges; mais cet attrait ne conduit pas toujours à une renonciation du français. En outre, en sortant de l'adolescence, le francophone tend à réaménager son rapport à sa langue de sorte qu'il la réaccueille en lui.

\section{Conclusion}

83. L'argument que j'ai développé dans ce document a pris ses assises sur une série de principes fortement liés les uns aux autres.

i. Dans les sociétés postmodernes, les médias constituent l'un des principaux facteurs de détermination de l'organisation sociale; sans eux, ces sociétés ne peuvent se reproduire, car leurs populations sont trop nombreuses, trop densifiées et trop diversifiées pour assurer aussi bien l'harmonie du tout que le respect des particularités.

ii. Les sociétés postmodernes sont formées de communautés dont le statut est relativement important parce qu'elles comptent bon nombre de membres et parce que l'histoire les met en position de force. Ces com-

Médias, langue et littératie en Ontario français, Sudbury, Centre franco-ontarien de ressources en alphabétisation, 1998. Au moment où nous écrivions cet affidavit, il n'existait, à notre connaissance, aucune autre donnée sur le rapport qu'entretiennent avec leurs médias les francophones du sud-ouest de la province et qui comparait leurs habitudes à celles des francophones des autres régions. 
munautés ont besoin de médias pour se faire être. En l'absence de ces médias, elles sont condamnées à ne recevoir et à ne faire circuler en elles que les messages qu'elles ne produisent pas ou qui ne leur sont pas destinés en propre. Cela rend à peu près impossible leur reproduction compte tenu de la place qu'occupent les médias dans la postmodernité.

iii. Les communautés qui composent les sociétés postmodernes sont normalement à la fois unes et divisibles. Les médias sont nécessaires pour assurer aussi bien l'unité de l'ensemble que la spécificité des sousensembles. Mais la reproduction des particularités des sous-ensembles n'est possible que si, effectivement, des messages médiatiques leur sont destinés dans cet esprit. Ces messages s'adressent alors aux individus du sous-ensemble en les informant des questions pratiques du milieu et en faisant connaître des événements et des personnages qui appartiennent au milieu et à la communauté. Si le sous-ensemble de la communauté ne trouve pas ces informations, alors il se tourne vers des messages médiatiques qui ne lui sont pas destinés en tant que tels, ce qui est d'autant plus probable que le milieu produit des messages pratiques pour d'autres que lui.

iv. Quand l'interaction de la démographie, de l'économie, de la culture et des médias ne rend pas possible la production de messages médiatiques pour une communauté ou pour un de ses sous-ensembles, alors il appartient à l'État d'intervenir en fonction de l'importance historique et démographique de la communauté ou de ses sous-ensembles.

v. Les médias, à eux seuls, ne peuvent assurer la reproduction d'une communauté ; leur contribution doit être rattachée à celle des autres institutions qui sont nécessaires à la vie en société. École, médias, travail, 
centre culturel, maison d'édition, bibliothèque, centre sportif sont nécessaires les uns aux autres.

84. Mon raisonnement a ensuite fait état de la situation francoontarienne et, plus généralement, de la position du minoritaire au Canada français.

vi. La minorité franco-ontarienne forme une communauté importante au sein de la province. Sur plusieurs plans, sa situation se compare à celle de la majorité. Mais elle n'en demeure pas moins une communauté dont le système institutionnel est grandement perfectible et dont l'imaginaire est envahi de symboliques proanglophones. On repère rapidement le signe de cet imaginaire dans les comportements linguistiques qui sont relatifs aux médias. Ces comportements, en effet, sont le témoignage d'une forte attirance de l'anglais.

vii. L'attrait de l'anglais est fort, en effet. Mais il n'abolit pas le recours au français; il en réduit la portée. Cette réduction est fortement attribuable à la place symbolique qu'occupe l'anglais dans l'imaginaire collectif, mais elle a aussi pour corollaire la disponibilité des médias. Or, dans une société hypermédiatisée comme le Canada, si le minoritaire ne trouve pas ce dont il a besoin sur le plan médiatique dans les messages qui lui sont destinés, alors il le trouvera dans les médias de la majorité. Et cela risque d'autant plus d'arriver que ces médias ont un contenu pratique, comme c'est souvent le cas des émissions de radio, notamment des programmations du matin.

85. Ma réflexion s'est terminée par une description du rapport aux médias de la minorité francophone du Sud-Ouest.

viii. Les Franco-Ontariens du Sud-Ouest ont beaucoup en partage avec les autres francophones de la province. Mais leur sur-minorisation les rend plus vulnérables que ceux des régions comme l'Est et le Nord-Est. La langue d'usage des médias, notamment chez les ado- 
lescents, en est le témoignage. Malgré tout, on trouve chez eux un rapport aux médias, chez les adultes, qui témoigne d'une nette demande de messages en français. Par conséquent, pour deux raisons divergentes, le Sud-Ouest de l'Ontario a besoin qu'on lui diffuse des messages en français, et dans la concrétude de la radio. La logique économie-culture-médias-démographie ne pouvant suffire à desservir la population, la radio d'État doit intervenir parce que :

- $\quad$ les jeunes ont besoin de messages en français parce qu'ils ont tendance à les négliger, ce qu'ils feront d'autant plus que leur environnement ne comptera pas de messages pertinents qui sont tout à fait attachés à leur vécu;

- $\quad$ les adultes ont aussi besoin de ces messages bien simplement parce qu'ils les réclament et que, faute de les trouver, ils devront syntoniser les stations de langue anglaise.

86. Et l'intervention de la radio d'État est, à mes yeux, d'autant plus requise :

$>\quad$ que la minorité franco-ontarienne, par sa francité, jouit d'un statut particulier dans la nation canadienne ;

$>$ que la population du Sud-Ouest de la province constitue un sous-ensemble significatif de cette minorité ;

$>\quad$ que les médias font partie du système médiatique dont a besoin une communauté pour se reproduire ;

$>\quad$ qu'en situation de concurrence des médias, l'absence de cette radio représente une menace réelle pour ce sous-ensemble ;

$>\quad$ que la radio, surtout par sa dimension pratique et spécifique, constitue l'un des principaux constituants de l'ensemble médiatique.

87. Cet affidavit est assermenté aux fins de la procédure citée ci-haut et pour aucun autre motif. 